\title{
Transverse-velocity fluctuations in a liquid under steady shear
}

\author{
José M. Ortiz de Zárate* and Jan V. Sengers ${ }^{\dagger}$ \\ Departamento de Física Aplicada I, Facultad de Física, Universidad Complutense, 28040 Madrid, Spain
}

(Received 14 November 2007; published 21 February 2008)

\begin{abstract}
We present an analysis of the transverse-velocity fluctuations in an isothermal liquid layer with a uniform shear rate between two parallel horizontal boundaries as a function of the wave number and the Reynolds number. The results were obtained by solving a stochastic version of the Orr-Sommerfeld equation subject to no-slip boundary conditions in a second-order Galerkin approximation. We find that the spatial Fourier transform of the transverse-velocity fluctuations exhibits a maximum as a function of the (horizontal) wave number $q_{\|}$. This maximum is associated with a crossover from a $q_{\|}^{-4}$ dependence for larger $q_{\|}$to a $q_{\|}^{2}$ dependence for small $q_{\|}$. The $q_{\|}^{-4}$ dependence at larger wave numbers is independent of the boundary conditions, but the small$q_{\|}$behavior is strongly affected by the boundary conditions. The nonequilibrium enhancement of the intensity of the transverse-velocity fluctuations remains finite for all values of the Reynolds number, but increases approximately with the square of the Reynolds number. The relation between our results and those obtained by previous authors in the absence of boundary conditions is elucidated.
\end{abstract}

DOI: 10.1103/PhysRevE.77.026306

PACS number(s): 47.15. $-\mathrm{x}, 05.20 . \mathrm{Jj}, 05.40 .-\mathrm{a}$

\section{INTRODUCTION}

During the past two decades it has become evident that thermally excited fluctuations in fluids in nonequilibrium steady states, even in the absence of convection or turbulence, are always spatially long ranged, as reviewed elsewhere [1-3]. As a consequence, thermal nonequilibrium fluctuations in fluids are affected by the presence of boundaries. In some previous publications [4,5], we have evaluated such finite-size effects on the static structure factor of liquid layers subjected to a stationary temperature gradient, sometimes referred to as the Rayleigh-Bénard problem. It is the purpose of this paper to consider the effect of the presence of boundaries on the velocity fluctuations in a liquid with uniform shear flow. Specifically, we shall consider the transversevelocity fluctuations that are most strongly affected by the presence of shear.

A number of previous investigators have studied fluctuations in sheared fluids in the absence of boundary conditions [6-12]. Several authors [6-8] have arrived at the conclusion that the nonequilibrium autocorrelation functions should decay as $r^{-1}$ with the distance $r$. Lutsko and Dufty [9-11] have argued that the autocorrelation functions should actually exhibit a crossover from an $r^{-1}$ decay to a stronger algebraic power-law decay for larger values of $r$. Specifically, the transverse-velocity autocorrelation function should exhibit a dependence on the distance $r$ proportional to $r^{-5 / 3}$ [11].

It turns out that not only the intensity of the fluctuations in sheared fluids but also the range of the correlation functions strongly depends on the direction of the wave vector of the fluctuations. It is well known that the long-ranged contribution that decays as $r^{-1}$ vanishes when the wave vector is in the direction of the fluid-velocity gradient. However, fluctuating hydrodynamics predicts this contribution to vanish also

\footnotetext{
*jmortizz@fis.ucm.es

†Permanent address: Institute for Physical Science and Technology, University of Maryland, College Park, MD 20742-8510, USA.
}

for fluctuations with a wave vector perpendicular to the fluid-velocity gradient $[7,9,10]$, in which direction one would expect the effect of the shear on the fluctuations to be significant. The particular case of velocity fluctuations with wave vector in the direction of the flow has been considered by Wada and Sasa [12], who concluded that the spatial spectrum of these velocity fluctuations should exhibit a crossover from a $q^{-4}$ dependence for larger wave numbers $q$ to a $q^{-4 / 3}$ dependence for small $q$.

Generally, fluctuations in fluids in steady nonequilibrium states can be obtained from fluctuating hydrodynamics with a local application of the fluctuation-dissipation theorem $[2,13]$. This approach was proposed by Ronis and Procaccia [14] as an alternative to the original derivation of Kirkpatrick et al. [15] for fluctuations in fluids subjected to a temperature gradient, and the validity of the extension of fluctuating hydrodynamics to nonequilibrium steady states has been confirmed experimentally [2]. We shall show that, for the case of transverse-velocity fluctuations in sheared liquids, the derivation can be greatly simplified by starting from a stochastic version of the Orr-Sommerfeld equation. The deterministic version of the Orr-Sommerfeld equation is frequently used in fluid mechanics in the analysis of planar Couette or Poiseuille flows $[16,17]$. Using this procedure without imposing boundary conditions, we shall not only recover expressions for the transverse-velocity fluctuations that are completely consistent with previous results obtained by Lutsko and Dufty [9-11], but also obtain an explicit expression for the velocity fluctuations with wave vector perpendicular to the fluid-velocity gradient in agreement with the predictions of Wada and Sasa [12]. The main point of our present paper is to show that boundary effects cause a pronounced modification of the wave-number dependence of the spatial Fourier transform of the velocity fluctuations.

We shall proceed as follows. In Sec. II we explain how a stochastic version of the Orr-Sommerfeld equation for the velocity fluctuations can be obtained from the full set of fluctuating hydrodynamics equations previously used by other authors. In Sec. III we present a simple procedure for solving this stochastic Orr-Sommerfeld equation in the ab- 
sence of any boundary conditions and show how this simple procedure reproduces all physical aspects of the velocity fluctuations noticed by previous investigators. In addition, this solution allows us to elucidate explicitly the anisotropic nature of the velocity fluctuations in a liquid under steady shear. In Sec. IV we study the modification of the velocity fluctuations due to the presence of boundaries. This is done by solving the stochastic Orr-Sommerfeld equation for the amplitude of the transverse-velocity fluctuations by taking into account the appropriate boundary conditions for the velocity fluctuations. In the presence of realistic boundary conditions, an exact solution would have to be obtained numerically. However, we are able to obtain explicit analytical expressions for the amplitude of the nonequilibrium velocity fluctuations as a function of the wave number by employing a second-order Galerkin approximation, which appears to yield an excellent approximation to the exact solution for the nonequilibrium fluctuations. We conclude the paper with some discussion of the physical meaning of the results in Sec. V.

\section{STOCHASTIC ORR-SOMMERFELD EQUATION}

We consider an isothermal liquid with a uniform density $\rho_{0}$ and with a mean velocity $\mathbf{v}_{0}=\left\{\dot{\gamma}_{0} z, 0,0\right\}$ in the $x$ direction, with $\dot{\gamma}_{0}$ representing a constant shear rate in the $z$ direction, so that the $x, y$, and $z$ coordinates represent the velocity, the vorticity, and the the velocity-gradient directions, respectively. In fluid mechanics this arrangement is referred to as the case of (isothermal) planar Couette flow. We note that some other investigators in the field have chosen the $z$ axis in the vorticity direction [6-12], but we adopt here the nomenclature of Drazin and Reid [16], sometimes referred to as the meteorological convention [18]. We prefer this convention to remain consistent with our previous analysis of finite-size effects in the Rayleigh-Bénard problem, where the temperature gradient was also taken to be in the $z$ direction [2-5]. Without loss of generality, we can assume the mean velocity to be in the positive $x$ direction for $z>0$ and in the negative $x$ direction for $z<0$ [16]. The fluctuations $\delta \rho(\mathbf{r}, t)$ and $\delta \mathbf{v}(\mathbf{r}, t)$ of the density and the velocity around their mean values $\rho_{0}$ and $\mathbf{v}_{0}$, respectively, depend on the location $\mathbf{r}$ and the time $t$. As documented in the literature, the spatialtemporal evaluation of the fluctuations satisfies fluctuatinghydrodynamics equations which can be linearized in fluctuating fields, if one assumes the fluctuations to be small [19]. For the present case one thus obtains for $\delta \rho(\mathbf{r}, t)$ and $\delta \mathbf{v}(\mathbf{r}, t)$ the following set of linearized fluctuating-hydrodynamics equations $[2,6-11]$ :

$$
\begin{aligned}
\partial_{t}(\delta \rho)+\dot{\gamma}_{0} z \partial_{x}(\delta \rho)+\rho_{0} \boldsymbol{\nabla} \cdot \delta \mathbf{v}=0, \\
\partial_{t}(\delta \mathbf{v})+\dot{\gamma}_{0} z \partial_{x}(\delta \mathbf{v})+\dot{\gamma}_{0} \delta v_{z} \hat{\mathbf{x}} \\
=\frac{-\nabla \delta \rho}{\rho_{0}^{2} \varkappa_{T}}+\nu \nabla^{2}(\delta \mathbf{v}) \\
\quad+\frac{1}{\rho_{0}}\left(\eta_{v}+\frac{\eta}{3}\right) \nabla(\boldsymbol{\nabla} \cdot \delta \mathbf{v})+\frac{1}{\rho_{0}} \nabla(\delta \Pi),
\end{aligned}
$$

where $\varkappa_{T}$ is the isothermal compressibility, $\eta$ the shear viscosity, $\eta_{v}$ the bulk viscosity, and $\nu=\eta / \rho_{0}$ the kinematic viscosity. We refer the reader to the relevant literature for further discussion of Eqs. (1), in particular to Eqs. (11.2) in Ref. [2].

The formulation of these fluctuating-hydrodynamics equations is completed with the specification of the correlation function among the various components of the random stress $\delta \Pi$, which is the only natural intrinsic source of stochastic forcing, usually referred to as thermal noise, in our isothermal problem. These correlation functions are given by the fluctuation-dissipation theorem (FDT), which for an isothermal fluid is given by $[2,19,20]$

$$
\begin{aligned}
& \left\langle\delta \Pi_{i j}(\mathbf{r}, t) \cdot \delta \Pi_{k l}\left(\mathbf{r}^{\prime}, t^{\prime}\right)\right\rangle \\
& =2 k_{B} T \delta\left(\mathbf{r}-\mathbf{r}^{\prime}\right) \delta\left(t-t^{\prime}\right)\left[\eta\left(\delta_{i k} \delta_{j l}+\delta_{i l} \delta_{j k}\right)\right. \\
& \left.\quad+\left(\eta_{v}-\frac{2}{3} \eta\right) \delta_{i j} \delta_{k l}\right]
\end{aligned}
$$

where $k_{B}$ is Boltzmann's constant and $T$ the average local temperature.

While several previous investigators [2,6-11] have considered the full set of fluctuating-hydrodynamics equations, we show how by a series of approximations Eqs. (1) can be reduced to a single stochastic partial differential equation (the stochastic Orr-Sommerfeld equation) for the transversevelocity fluctuations. This formulation represents some simplification, but will turn out to capture all essential physical features of the fluctuations in a liquid under steady shear flow. The approximations are identical to those adopted in the fluid-dynamics literature in the derivation of the deterministic version of the Orr-Sommerfeld equation [16].

Our main hydrodynamic approximation is that we are dealing with divergence-free flow. As explained by Landau and Lifshitz [19], this assumption is justified as long as the fluid flow velocity is much smaller than the sound velocity, which is certainly the case for flow velocities encountered in laminar Couette flow. This simplification is more conveniently implemented if we switch from density to pressure fluctuations, $\delta p(\mathbf{r}, t)=-1 /\left(\rho_{0} \varkappa_{T}\right) \delta \rho(\mathbf{r}, t)$, as the relevant fluctuating variable. Then, instead of (1), we obtain

$$
\begin{aligned}
\varkappa_{T} \partial_{t}(\delta p)+\varkappa_{T} \dot{\gamma}_{0} z \partial_{x}(\delta p)+\nabla \cdot \delta \mathbf{v}=0, \\
\partial_{t}(\delta \mathbf{v})+\dot{\gamma}_{0} z \partial_{x}(\delta \mathbf{v})+\dot{\gamma}_{0} \delta v_{z} \hat{\mathbf{x}} \\
=-\frac{\nabla \delta p}{\rho_{0}}+\nu \nabla^{2}(\delta \mathbf{v})+\frac{1}{\rho_{0}}\left(\eta_{v}+\frac{\eta}{3}\right) \nabla(\boldsymbol{\nabla} \cdot \delta \mathbf{v}) \\
+\frac{1}{\rho_{0}} \nabla(\delta \Pi) .
\end{aligned}
$$

Next, as usual in the fluid-dynamics literature [16], we express the working equations in dimensionless variables, defined in terms of the half height $L$ of the liquid layer, the shear rate $\dot{\gamma}_{0}$, and the density $\rho_{0}$ :

$$
\widetilde{\mathbf{r}}=\frac{\mathbf{r}}{L}, \quad \tilde{t}=\dot{\gamma}_{0} t, \quad \delta \widetilde{p}=\frac{\delta p}{\rho_{0} L^{2} \dot{\gamma}_{0}^{2}},
$$




$$
\delta \widetilde{\mathbf{v}}=\frac{\delta \mathbf{v}}{L \dot{\gamma}_{0}}, \quad \delta \widetilde{\Pi}=\frac{\delta \Pi}{\rho_{0} L^{2} \dot{\gamma}_{0}^{2}} .
$$

In terms of dimensionless variables we thus obtain

$$
\begin{gathered}
\epsilon \operatorname{Re}^{2}\left[\partial_{t}(\delta p)+z \partial_{x}(\delta p)\right]+\nabla \cdot \delta \mathbf{v}=0 \\
\partial_{t}(\delta \mathbf{v})+z \partial_{x}(\delta \mathbf{v})+\delta v_{z} \hat{\mathbf{x}}= \\
+\nabla \delta p+\frac{1}{\operatorname{Re}} \nabla^{2}(\delta \mathbf{v})+\frac{1}{R_{v}} \nabla(\nabla \cdot \delta \mathbf{v}) \\
+\nabla(\Pi),
\end{gathered}
$$

where the Reynolds and the other dimensionless numbers appearing above are given by

$$
\operatorname{Re}=\frac{\dot{\gamma}_{0} L^{2}}{\nu}, \quad R_{v}=\frac{\rho_{0} \dot{\gamma}_{0} L^{2}}{\left(\eta_{v}+\frac{\eta}{3}\right)}, \quad \epsilon=\rho_{0} \varkappa_{T} \nu^{2} L .
$$

Notice that in Eq. (5) we have dropped the tildes in the notation for the various fields, since in the remainder of this paper we shall always use dimensionless variables. Next, we take the limit $\epsilon \rightarrow 0$ for a fluid with uniform density $\rho$ in the set of equations (5), as is usually done in the literature on hydrodynamic stability [16]. Hence, the dimensionless fluctuating-hydrodynamics equations we shall consider in this paper are

$$
\begin{gathered}
\boldsymbol{\nabla} \cdot \delta \mathbf{v}=0, \\
\partial_{t}(\delta \mathbf{v})+z \partial_{x}(\delta \mathbf{v})+\delta v_{z} \hat{\mathbf{x}}=-\nabla \delta p+\frac{1}{\operatorname{Re}} \nabla^{2}(\delta \mathbf{v})+\nabla(\delta \Pi) .
\end{gathered}
$$

To further simplify the system of differential equations (7a) and (7b), we take a double rotational in Eq. (7b), so as to eliminate the pressure fluctuations from the problem. Then, by using Eq. (7a), we obtain a single closed equation for the vertical velocity component $\delta v_{z}(\mathbf{r}, t)$, to which we refer as the stochastic Orr-Sommerfeld equation

$$
\partial_{t}\left(\nabla^{2} \delta v_{z}\right)+z \partial_{x}\left(\nabla^{2} \delta v_{z}\right)-\frac{1}{\operatorname{Re}} \nabla^{4}\left(\delta v_{z}\right)=\{\boldsymbol{\nabla} \times \boldsymbol{\nabla} \times[\nabla(\delta \Pi)]\}_{z} .
$$

The stochastic Orr-Sommerfeld equation (8) will be the starting point for our evaluation of the nonequilibrium transverse-velocity fluctuations. The specification of the random-noise term on the right-hand side (RHS) can be readily obtained from Eq. (2), as discussed below.

In the case of a liquid subjected to a stationary temperature gradient, the long-ranged nonequilibrium fluctuations are caused by a coupling of the temperature fluctuations with the transverse-velocity fluctuations through the temperature gradient. Hence, to determine these nonequilibrium fluctuations one needs to solve two partial differential equations simultaneously $[2,21]$. However, as pointed out by Lutsko and Dufty $[9,10]$, the long-ranged nonequilibrium fluctuations in a liquid under steady shear are caused not by a coupling of two different hydrodynamic modes with the same wave vector, but by a coupling of viscous modes with differ- ent wave vectors through the velocity gradient. Hence, in the present case the fluctuations of the velocity in the direction of the shear gradient can be determined from a single partial differential equation, namely, Eq. (8).

Of course, for a determination of the fluctuations of all three components of the velocity, additional information will be needed. The appropriate additional partial differential equation, complementing Eq. (8), can be obtained by taking a single curl of Eq. (7b), so as to obtain an equation for the component of the vorticity fluctuations parallel to the shear gradient $\delta \omega_{z}=\partial_{y} \delta v_{x}-\partial_{x} \delta v_{y}$ :

$$
\partial_{t}\left(\delta \omega_{z}\right)+z \partial_{x}\left(\delta \omega_{z}\right)-\partial_{y} \delta v_{z}-\frac{1}{\operatorname{Re}} \nabla^{2}\left(\delta \omega_{z}\right)=\{\boldsymbol{\nabla} \times[\nabla(\delta \Pi)]\}_{z} .
$$

Equations (8) and (9) form a pair of coupled stochastic differential equations that would need to be solved simultaneously for the fluctuating fields $\delta v_{z}$ and $\delta \omega_{z}$ with appropriate boundary conditions. The deterministic version of Eq. (9) is commonly referred to in the fluid-mechanics literature as the Squire equation [17]. The random-noise terms on the RHS of the stochastic Orr-Sommerfeld Eq. (8) and the stochastic Squire equation (9) act as additive thermal noise, whose correlation functions immediately follow from the FDT for the random stress, given by Eq. (2). However, to elucidate the physical nature of the transverse-velocity fluctuations, use of the single partial differential equation (8) will suffice.

\section{NONEQUILIBRIUM VELOCITY FLUCTUATIONS IN THE ABSENCE OF BOUNDARY CONDITIONS}

The fluid mean velocity cannot grow indefinitely in the positive and negative $z$ directions, and in reality the liquid layer is confined between two horizontal boundaries. However, to make contact with the work of previous investigators, we shall first solve Eq. (8) for the velocity fluctuations without accounting for any boundary conditions. As usual in the derivation of so-called bulk nonequilibrium fluctuations [2], we apply a full spatiotemporal Fourier transform to the appropriate stochastic differential equation, which in the present case is Eq. (8). We thus obtain

$$
q^{2}\left(i \omega+\frac{q^{2}}{\operatorname{Re}}\right) \delta v_{z}(\omega, \mathbf{q})-q_{x} \frac{\partial}{\partial q_{z}}\left[q^{2} \delta v_{z}(\omega, \mathbf{q})\right]=F_{z}(\omega, \mathbf{q}),
$$

where $\omega$ and $\mathbf{q}$ are the frequency and the wave vector of the fluctuations, respectively, while $F_{z}(\omega, \mathbf{q})$ represents a random noise term that corresponds to (minus) the Fourier transform of the combination of derivatives of the random stress that appears on the RHS of Eq. (8). Next, to solve Eq. (10) for $\delta v_{z}(\omega, \mathbf{q})$, we adopt the approach of Lutsko and Dufty [9-11], and express the solution in terms of a Green's function:

$$
\delta v_{z}\left(\omega, \mathbf{q}_{\|}, q_{z}\right)=\int_{-\infty}^{\infty} d u G\left(\omega, \mathbf{q}_{\|}, q_{z}, u\right) F_{z}\left(\omega, \mathbf{q}_{\|}, u\right),
$$

where the Green's function $G\left(\omega, \mathbf{q}_{\|}, q_{z}, u\right)$ is the solution of 


$$
\begin{aligned}
& q^{2}\left(i \omega+\frac{q^{2}}{\operatorname{Re}}\right) G\left(\omega, \mathbf{q}_{\|}, q_{z}, u\right)-q_{x} \frac{\partial}{\partial q_{z}}\left[q^{2} G\left(\omega, \mathbf{q}_{\|}, q_{z}, u\right)\right] \\
& \quad=\delta\left(q_{z}-u\right),
\end{aligned}
$$

with $\mathbf{q}_{\|}=\left\{q_{x}, q_{y}\right\}$ representing the projection of the wave vector $\mathbf{q}$ of the fluctuations onto the plane perpendicular to the shear gradient, i.e., onto the horizontal $x y$ plane.

To evaluate the autocorrelation function of the verticalvelocity fluctuations we assume that the fluctuationdissipation theorem (2), originally derived for fluctuations in a fluid in equilibrium, continues to hold for nonequilibrium steady states with the physical properties to be taken at their mean values. The validity of this assumption has been verified experimentally for the case of the Rayleigh-Bénard problem [2]. As already anticipated, the autocorrelation $\left\langle F_{z}^{*}(\omega, \mathbf{q}) F_{z}\left(\omega^{\prime}, \mathbf{q}^{\prime}\right)\right\rangle$ can be easily obtained from the relationship of $F_{z}(\mathbf{r}, t)$ with the random stress and from the FDT (2); see Refs. $[2,4,5]$. To simplify this calculation it is convenient to employ the divergence-free approximation (7a), which allows us to effectively neglect the term proportional to the bulk viscosity in the FDT (2); see Ref. [2]. In terms of the dimensionless variables used in this paper,

$$
\frac{\left\langle F_{z}^{*}(\omega, \mathbf{q}) F_{z}\left(\omega^{\prime}, \mathbf{q}^{\prime}\right)\right\rangle}{(2 \pi)^{4} 2 \tilde{S}}=q_{\|}^{2} q^{4} \delta\left(\omega-\omega^{\prime}\right) \delta\left(\mathbf{q}-\mathbf{q}^{\prime}\right),
$$

where the dimensionless strength $\widetilde{S}$ of the thermal noise is given by

$$
\widetilde{S}=\frac{k_{B} T}{\dot{\gamma}_{0}^{3} L^{7}} \frac{\nu}{\rho_{0}}=\frac{k_{B} T}{\rho_{0} L^{3}} \frac{1}{\dot{\gamma}_{0}^{2} L^{2}} \frac{1}{\operatorname{Re}} .
$$

Next, from Eqs. (11) and (13), we readily evaluate the autocorrelation function of the vertical-velocity fluctuations. It can be expressed in the form

$$
\begin{aligned}
\left\langle\delta v_{z}^{*}(\omega, \mathbf{q}) \delta v_{z}\left(\omega^{\prime}, \mathbf{q}^{\prime}\right)\right\rangle= & C_{z z}\left(\omega, \mathbf{q}_{\|}, q_{z}, q_{z}^{\prime}\right)(2 \pi)^{3} \delta\left(\omega-\omega^{\prime}\right) \\
& \times \delta\left(\mathbf{q}_{\|}-\mathbf{q}_{\|}^{\prime}\right),
\end{aligned}
$$

where

$$
C_{z z}\left(q_{z}, q_{z}^{\prime}\right)=4 \pi \widetilde{S} q_{\|}^{2} \int_{-\infty}^{\infty}\left(q_{\|}^{2}+u^{2}\right)^{2} G^{*}\left(q_{z}, u\right) G\left(q_{z}^{\prime}, u\right) d u .
$$

Here, to simplify the notation, we dropped the implicit $\omega, \mathbf{q}_{\|}$ dependence of all quantities appearing in Eq. (16), retaining explicitly only the $q_{z}$ dependence (or $q_{z}$ and $q_{z}^{\prime}$ for the Green's function). To proceed with the calculation of the velocity autocorrelation function, we need first to discuss the explicit form of the Green's function.

\section{A. Explicit form of the Green's function}

We can solve Eq. (12) for $G\left(\omega, \mathbf{q}_{\|}, q_{z}, u\right)$, to obtain from it explicit expressions for the autocorrelation function of velocity fluctuations parallel to the shear gradient. Since it is a linear equation, the procedure is simple and, at least formally, the Green's function can be expressed as

$$
G\left(q_{z}, u\right)=\frac{1}{q_{\|}^{2}+q_{z}^{2}} \int_{0}^{\infty} \exp \left[-\Omega\left(q_{z}, \alpha\right)\right] \delta\left(q_{z}-u+q_{x} \alpha\right) d \alpha
$$

with

$$
\Omega\left(q_{z}, \alpha\right)=\frac{q_{x}^{2}}{3 \operatorname{Re}} \alpha^{3}+\frac{q_{z} q_{x}}{\operatorname{Re}} \alpha^{2}+\left(i \omega+\frac{q_{\|}^{2}+q_{z}^{2}}{\operatorname{Re}}\right) \alpha .
$$

Equations (17) and (18) are valid for both positive and negative values of $q_{x}$.

At this point, it is worthwhile to digress a little bit to discuss a technical issue. In principle one can add to Eq. (17) for the Green's function any solution of the homogeneous problem corresponding to Eq. (12). However, the actual expression (17) is the "correct" Green's function in the sense that, when $q_{x} \rightarrow 0$, it reduces to the well-known equilibrium Green's function [22-24], namely,

$$
G\left(q_{z}, u\right) \stackrel{q_{x} \rightarrow 0}{\rightarrow} \frac{1}{q_{\|}^{2}+q_{z}^{2}} \frac{\delta\left(q_{z}-u\right)}{i \omega+\frac{q_{\|}^{2}+q_{z}^{2}}{\operatorname{Re}}},
$$

while the addition of any particular solution of the homogeneous problem to (17) would not yield the correct asymptotic behavior given by Eq. (19).

We now continue our calculation by substituting Eq. (17) into Eq. (16) and then integrating it over the variable $u$, so as to obtain

$$
\begin{aligned}
C_{z z}\left(q_{z}, q_{z}^{\prime}\right)= & \frac{4 \pi \tilde{S} q_{\|}^{2}}{\left(q_{\|}^{2}+q_{z}^{2}\right)\left(q_{\|}^{2}+q_{z}^{\prime 2}\right)} \int_{0}^{\infty} d \beta \int_{0}^{\infty} d \alpha\left[q_{\|}^{2}\right. \\
& \left.+\left(q_{z}^{\prime}+q_{x} \beta\right)^{2}\right]^{2} \delta\left(q_{x} \alpha-q_{x} \beta+q_{z}-q_{z}^{\prime}\right) \\
& \times \exp \left[-\Omega^{*}\left(q_{z}, \alpha\right)-\Omega\left(q_{z}^{\prime}, \beta\right)\right] .
\end{aligned}
$$

Some simple algebra yields

$$
\begin{aligned}
\Omega^{*}\left(q_{z}, \alpha\right)+\Omega\left(q_{z}^{\prime}, \beta\right) & \\
= & -i \omega(\alpha-\beta)+\frac{1}{\operatorname{Re}}\left(\frac{q_{x}^{2}}{3}\left(\alpha^{3}+\beta^{3}\right)\right. \\
& \left.\quad+q_{x}\left(q_{z} \alpha^{2}+q_{z}^{\prime} \beta^{2}\right)+q_{\|}^{2}(\alpha+\beta)+\alpha q_{z}^{2}+\beta q_{z}^{\prime 2}\right) .
\end{aligned}
$$

We could investigate the dependence of the velocity autocorrelation function by applying to Eq. (20) a Fourier (anti) transform in the frequency $\omega$. Such an investigation was pursued by Lutsko and Dufty [9,11], who demonstrated that this time correlation function has an interesting nonexponential behavior. However, we are specifically interested in the Fourier-transformed equal-time correlation function, which determines the intensity of the fluctuations and which can be related to what has been called energy amplification [25] in the fluid-mechanics literature. As is well known, this correlation function is proportional to the integral over the frequency of $C_{z z}\left(\omega, \mathbf{q}_{\|}, q_{z}, q_{z}^{\prime}\right)$. In view of the simple $\omega$ depen- 
dence of Eq. (21), the integral over the frequency of (20) can be readily performed, resulting in

$$
\frac{1}{2 \pi} \int_{-\infty}^{\infty} d \omega C_{z z}\left(\omega, \mathbf{q}_{\|}, q_{z}, q_{z}^{\prime}\right)=C_{z z}(\mathbf{q}) 2 \pi \delta\left(q_{z}-q_{z}^{\prime}\right)
$$

with

$$
\begin{aligned}
C_{z z}(\mathbf{q})= & 2 \tilde{S} \frac{q_{\|}^{2}}{q^{4}} \int_{0}^{\infty} d \beta\left[q_{\|}^{2}+\left(q_{z}+q_{x} \beta\right)^{2}\right]^{2} \\
& \times \exp \left(\frac{-2 \beta}{3 \operatorname{Re}}\left(q_{x}^{2} \beta^{2}+3 \beta q_{x} q_{z}+3 q^{2}\right)\right),
\end{aligned}
$$

in complete agreement with Wada and Sasa [12]. The fact that the variables $\alpha$ and $\beta$ are always positive has been used to deduce Eq. (23).

A simple integration by parts of Eq. (23) allows us to display the intensity of fluctuations in the nonequilibrium steady state as a sum of the equilibrium intensity and a nonequilibrium enhancement, namely,

$$
C_{z z}(\mathbf{q})=C_{z z}^{(\mathrm{E})}(\mathbf{q})\left\{1+\widetilde{C}_{z z}^{(\mathrm{NE})}(\mathbf{q})\right\}
$$

where

$$
C_{z z}^{(\mathrm{E})}(\mathbf{q})=\tilde{S} \operatorname{Re} \frac{q_{\|}^{2}}{q^{2}},
$$

and

$$
\begin{aligned}
\tilde{C}_{z z}^{(\mathrm{NE})}(\mathbf{q})= & \frac{2 q_{x}}{q^{2}} \int_{0}^{\infty} d \beta\left(q_{z}+q_{x} \beta\right) \\
& \times \exp \left(\frac{-2 \beta}{3 \operatorname{Re}}\left(q_{x}^{2} \beta^{2}+3 \beta q_{x} q_{z}+3 q^{2}\right)\right) .
\end{aligned}
$$

Expression (25), of course, agrees with the equilibrium result as discussed at length in several textbooks [22-24]. For such a comparison we remind the reader that, when the noise is thermal, its intensity is given by Eq. (14), so that the product $\widetilde{S} \operatorname{Re}$ is independent of the Reynolds number and proportional only to $\left(\dot{\gamma}_{0} L\right)^{-2}$, which is the unit by which we have made the actual correlation function dimensionless in accordance with Eq. (4).

Expression (24) has the typical structure of nonequilibrium fluctuations. Expressions similar to Eq. (24) have been obtained for several other nonequilibrium states, like a stationary temperature gradient in one-component fluids [15] or a stationary concentration gradient in fluid mixtures [26]. Nowadays it is believed that the feature exhibited by Eq. (24), i.e., an enhancement of fluctuations, is a generic property of nonequilibrium steady states [27], even for systems that are far from any hydrodynamic instability. The physical origin of the nonequilibrium enhancement of thermal fluctuations can be traced to coupling between hydrodynamic modes caused by the presence of a gradient. In the particular case considered here, the nonequilibrium coupling arises between velocity fluctuations with different values of the $q_{z}$ component of the wave vector, as follows from the presence in Eq. (10) of a derivative with respect to this variable $[9,10]$.
The integral (26) cannot be evaluated analytically. However, it is very simple to obtain the asymptotic expansions for large and small wave numbers $q$. To specify these limits we introduce spherical coordinates by substituting $q_{z}=q \cos \theta$, $q_{\|}=q \sin \theta$, and $q_{x}=q \sin \theta \cos \phi$ into Eq. (26). After these substitutions, the two limits mentioned above can be properly taken, and we obtain

$$
\begin{gathered}
\widetilde{C}_{z z}^{(\mathrm{NE})}(\mathbf{q}) \rightarrow\left(\frac{2}{3}\right)^{1 / 3} \Gamma\left(\frac{2}{3}\right)(\sin \theta \cos \phi)^{2 / 3} \frac{\mathrm{Re}^{2 / 3}}{q^{4 / 3}}, \quad(27 \mathrm{a}) \\
\widetilde{C}_{z z}^{(\mathrm{NE})}(\mathbf{q}) \rightarrow \frac{q \rightarrow \infty}{2 q^{2}} \cos \phi \sin 2 \theta-\frac{\mathrm{Re}^{2}}{2 q^{4}} \cos ^{2} \phi \cos 2 \theta \sin ^{2} \theta,
\end{gathered}
$$

in agreement with the limiting expressions obtained by Wada and Sasa [12] for the particular case of fluctuations with wave vector $\mathbf{q}$ in the direction of the flow $(\theta=\pi / 2, \phi=0)$. Numerical computation of the full nonequilibrium enhancement given by Eq. (26) show that it represents a simple crossover between the two asymptotic behaviors displayed in Eqs. (27a) and (27b), and does not exhibit any intermediate structure. A crossover wave number $q_{\mathrm{co}}$ may be defined from the intercept of the two first terms in the asymptotic behaviors described by Eqs. (27a) and (27b), given by

$$
q_{\mathrm{co}}=\frac{\sqrt{3} \cos \theta}{2\left[\Gamma\left(\frac{2}{3}\right)\right]^{3 / 2}} \sqrt{|\sin 2 \theta \cos \phi|} \sqrt{\operatorname{Re}}
$$

Hence, the crossover from a $q^{-2}$ to a $q^{-4 / 3}$ wave-number dependence of the nonequilibrium enhancement of the fluctuations occurs at a value $q_{\mathrm{co}}$ of the wave number that is proportional to the square root of the Reynolds number, in agreement with an observation of Lutsko and Dufty [11], and confirmed by Wada and Sasa [12].

From Eqs. (27a) and (27b) we conclude that we have reproduced from the stochastic Orr-Sommerfeld equation the prediction of Machta et al. [6] and of Tremblay et al. [7] that $C_{z z}(q) \propto q^{-2}$ (corresponding to $r^{-1}$ dependence of the correlation function in real space), as well as a crossover to $C_{z z}(q) \propto q^{-4 / 3}$ (corresponding to an $r^{-5 / 3}$ dependence of the correlation function in real space), as suggested by Lutsko and Dufty [11].

However, not only the intensity of the nonequilibrium fluctuations but also the range of the fluctuations is strongly anisotropic. First of all, it is evident that the nonequilibrium enhancement of the fluctuations vanishes when $\theta=0$, i.e., when the wave vector is in the direction of the fluid-velocity gradient. Furthermore, it should be noticed that the first term in Eq. (27b) also vanishes when $\theta=\pi / 2$, i.e., when the wave vector is perpendicular to the velocity gradient. Therefore there exists a competition between a $q^{-2}$ and a $q^{-4}$ wavenumber dependence of the amplitude of the fluctuations for larger $q$, which depends on the angle $\theta$. When the wave vector is not perpendicular to the velocity gradient, the shear affects the transverse-velocity fluctuations only partially. Only for wave vectors perpendicular to the velocity gradient, i.e., when $\mathbf{q}=\mathbf{q}_{\|}$(or $q_{z}=0$ ) can the fluctuations of the vertical velocity $\delta v_{z}$ be fully identified with the fluctuations of the 


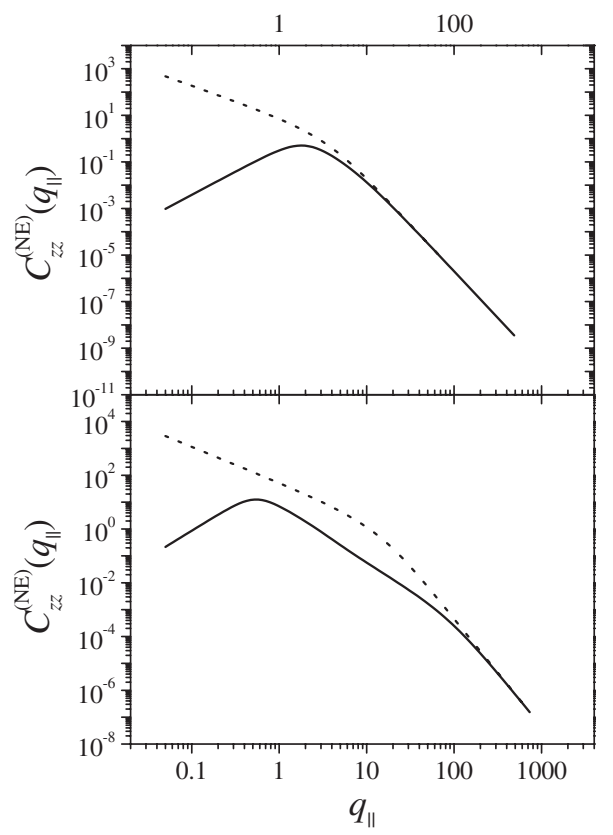

FIG. 1. Nonequilibrium enhancement of the transverse-velocity fluctuations as a function of the magnitude $q_{\|}$of the wave vector $\mathbf{q}_{\|}$ in the direction of the flow. Top panel is for $\mathrm{Re}=20$ and the bottom panel for $\mathrm{Re}=300$. The dashed curves represent the enhancement in the absence of any boundary conditions. The solid curves represent the enhancement that includes the effect of boundary conditions. For large $q_{\|}$the nonequilibrium enhancement becomes independent of the boundary conditions and the two curves coincide.

transverse velocity. These transverse-velocity fluctuations exhibit a crossover not from a $q^{-2}$ to a $q^{-4 / 3}$, but from a $q^{-4}$ to a $q^{-4 / 3}$ wave-number dependence, as previously noticed by Wada and Sasa [12]. This behavior is represented by the dashed curves in Fig. 1 for two values of the Reynolds number. The fact that these transverse-velocity fluctuations for larger $q$ vary with $q^{-4}$, just like the thermal fluctuations in fluids subjected to a stationary temperature gradient $[15,21]$, means that the spatial range of these correlations is much longer than correlations with a $r^{-1}$ decay, as discussed in previous publications [4,28].

\section{NONEQUILIBRIUM VELOCITY FLUCTUATIONS IN THE PRESENCE OF BOUNDARY CONDITIONS}

Planar Couette flow cannot be maintained without two bounding parallel plates that actually drive the fluid. Our previous experience with liquid layers subjected to a temperature gradient $[5,29]$ has shown that the presence of boundaries may dramatically affect the spatial spectrum of nonequilibrium fluctuations. Encouraged by these previous studies we investigate here the effects of confinement on fluctuations in a liquid under steady shear. For this purpose we consider no-slip boundary conditions at the two bounding plates located at $z= \pm 1$; thus

$$
\delta v_{z}=\partial_{z} \delta v_{z}=0 \quad \text { at } z= \pm 1 \text {. }
$$

We remind the reader that we continue to use dimensionless lengths scaled by the half height of the liquid layer, in accordance with Eqs. (4).
Our problem in this section is, again, to solve the stochastic differential equation Eq. (8), but subjected to the boundary conditions (29) at $z= \pm 1$. To accommodate the boundary conditions, as usual $[2,30]$, we now apply a Fourier transform in time and only in the plane perpendicular to the shear gradient, arriving at

$$
\begin{aligned}
& i\left(\omega+z q_{x}\right)\left(\partial_{z}^{2}-q_{\|}^{2}\right) \delta v_{z}\left(\omega, \mathbf{q}_{\|}, z\right)-\frac{1}{\operatorname{Re}}\left(\partial_{z}^{2}-q_{\|}^{2}\right)^{2} \delta v_{z}\left(\omega, \mathbf{q}_{\|}, z\right) \\
& \quad=F_{z}\left(\omega, \mathbf{q}_{\|}, z\right)
\end{aligned}
$$

where the stochastic forcing term $F_{z}\left(\omega, \mathbf{q}_{\|}, z\right)$ is the (partial) Fourier transform of the combination of derivatives of the stress tensor appearing on the RHS of Eq. (8).

In principle, one can solve Eq. (30) for $\delta v_{z}\left(\omega, \mathbf{q}_{\|}, z\right)$ subjected to the boundary conditions (29) by expanding the solution in a suitable set of eigenfunctions of the differential operator in the LHS of Eq. (30), as we have done previously for the case of the Rayleigh-Bénard problem, for both slipfree [4] and stick [2] boundary conditions. In the case of planar Couette flow, the eigenfunctions of the hydrodynamic operator (30) can be conveniently expressed in terms of Airy functions [31]. We plan to address this issue in a future presentation. However, for the sake of simplicity, and also to focus on the most relevant physical features of the problem under consideration, we adopt here a simpler approach by adopting a Galerkin approximation. The idea is to express the solution to (30) in terms of a series:

$$
\delta v_{z}\left(\omega, \mathbf{q}_{\|}, z\right)=\left(z^{2}-1\right)^{2} \sum_{N=0}^{\infty} A_{N}\left(\omega, \mathbf{q}_{\|}\right) z^{N} .
$$

This expression for $\delta v_{z}\left(\omega, \mathbf{q}_{\|}, z\right)$ satisfies the boundary conditions (29) for any set of coefficients $A_{N}\left(\omega, \mathbf{q}_{\|}\right)$. The polynomial $\left(z^{2}-1\right)^{2}$ that forces $\delta v_{z}\left(\omega, \mathbf{q}_{\|}, z\right)$ to satisfy the boundary conditions is usually referred to as the Galerkin test function. The coefficients $A_{N}\left(\omega, \mathbf{q}_{\|}\right)$are determined by projecting the stochastic differential equation (30) onto the elements of the polynomial basis Eq. (31) used to express $\delta v_{z}$. This requires solving the system of algebraic equations

$$
\sum_{M} X_{N M}\left(\omega, \mathbf{q}_{\|}\right) A_{M}\left(\omega, \mathbf{q}_{\|}\right)=F_{N}\left(\omega, \mathbf{q}_{\|}\right)
$$

where

$$
\begin{gathered}
X_{N M}\left(\omega, \mathbf{q}_{\|}\right)=\int_{-1}^{1} d z\left(z^{2}-1\right)^{2} z^{N}\left[\left(i\left(\omega+z q_{x}\right)\left(\partial_{z}^{2}-q_{\|}^{2}\right)\right.\right. \\
\left.\left.-\frac{1}{\operatorname{Re}}\left(\partial_{z}^{2}-q_{\|}^{2}\right)^{2}\right)\left(z^{2}-1\right)^{2} z^{M}\right] \\
F_{N}\left(\omega, \mathbf{q}_{\|}\right)=\int_{-1}^{1} d z\left(z^{2}-1\right)^{2} z^{N} F_{z}\left(\omega, \mathbf{q}_{\|}, z\right)
\end{gathered}
$$

Of course, such a Galerkin approximation is practical only if the series (31) can be truncated after a few terms, so that the algebraic set of equations (32) has a low enough dimensionality to be readily inverted and solved for $A_{N}\left(\omega, \mathbf{q}_{\|}\right)$. In this paper we shall keep the first two terms in the series (31), so 
as to obtain simple analytical solutions. We have checked that inclusion of more terms does not change our final result for the intensity of nonequilibrium velocity fluctuations parallel to the shear gradient (see below) by more than a few percent. Furthermore, our determination of the exact (numerical) solution confirms that the simple Galerkin approximation presented here represents qualitatively the exact solution for the nonequilibrium fluctuations very well, while differing quantitatively by just a few percent.

In addition, we have also made calculations with other test functions, like a combination of trigonometric functions or Chandrasekhar's functions, and obtained similar results with only very small quantitative differences. We have thus concluded that if the polynomial $\left(z^{2}-1\right)^{2}$ is chosen as the
Galerkin test function a second-order approximation in accordance with Eq. (31) is sufficient to elucidate the effects of the boundary conditions on the wave-number dependence of the nonequilibrium enhancement of the transverse-velocity fluctuations. The advantage of using the first two terms in the test-function representation given by Eq. (31) is its simplicity. We may also mention that in previous publications $[2,4,32]$ we have obtained Galerkin approximations for the fluctuations in confined layers of liquids and liquid mixtures subjected to a temperature gradient, with excellent results.

If we truncate the series (31), retaining only the first two terms, we obtain for the matrix appearing on the LHS of Eq. (32)

$$
X\left(\omega, \mathbf{q}_{\|}\right)=\frac{-256}{3465}\left[\begin{array}{cc}
11\left(i \omega\left(q_{\|}^{2}+3\right)+\frac{2 q_{\|}^{4}+12 q_{\|}^{2}+63}{2 \operatorname{Re}}\right) & i q_{x}\left(q_{\|}^{2}+11\right) \\
i q_{x} q_{\|}^{2} & i \omega\left(q_{\|}^{2}+11\right)+\frac{2 q_{\|}^{4}+44 q_{\|}^{2}+495}{2 \operatorname{Re}}
\end{array}\right],
$$

which can be easily inverted.

Our goal in the present section is to evaluate the autocorrelation function $\left\langle\delta v_{z}^{*}\left(\omega, \mathbf{q}_{\|}, z\right) \delta v_{z}\left(\omega^{\prime}, \mathbf{q}_{\|}^{\prime}, z^{\prime}\right)\right\rangle$. In view of the representation (31) of $\delta v_{z}\left(\omega, \mathbf{q}_{\|}, z\right)$, the solution will depend on the correlation functions $\left\langle A_{N}^{*}\left(\omega, \mathbf{q}_{\|}\right) A_{M}\left(\omega^{\prime}, \mathbf{q}_{\|}^{\prime}\right)\right\rangle$, which by inverting Eq. (32) can be evaluated from the projection of the random noise over the Galerkin polynomials Eq. (33b). Combining the fluctuation-dissipation theorem for the random stress with the definition (33b) of $F_{N}^{*}\left(\omega, \mathbf{q}_{\|}\right)$, as was done in previous publications dealing with fluctuations in the Rayleigh-Bénard problem [2,5,32], we obtain

$\left\langle F_{N}^{*}\left(\omega, \mathbf{q}_{\|}\right) F_{M}\left(\omega^{\prime}, \mathbf{q}_{\|}^{\prime}\right)\right\rangle=(2 \pi)^{3} 2 \widetilde{S} C_{N M}\left(\mathbf{q}_{\|}\right) \delta\left(\omega-\omega^{\prime}\right) \delta\left(\mathbf{q}_{\|}-\mathbf{q}_{\|}^{\prime}\right)$,

where, as in Eq. (14), $\widetilde{S}$ again represents the dimensionless amplitude of the thermal noise. The correlation matrix $C_{N M}\left(\mathbf{q}_{\|}\right)$can be easily obtained by exactly following the procedure outlined in some of our previous publications $[2,5,32]$. This procedure yields for the correlation matrix in Eq. (35)

$$
C\left(\mathbf{q}_{\|}\right)=\frac{128 q_{\|}^{2}}{315}\left[\begin{array}{cc}
2 q_{\|}^{4}+12 q_{\|}^{2}+63 & 0 \\
0 & \frac{2 q_{\|}^{4}+44 q_{\|}^{2}+495}{11}
\end{array}\right] .
$$

Next, combining Eqs. (31) and (35) with the inverse of Eq. (32), we immediately obtain for the autocorrelation function of interest

$$
\begin{aligned}
\left\langle\delta v_{z}^{*}\left(\omega, \mathbf{q}_{\|}, z\right) \delta v_{z}\left(\omega^{\prime}, \mathbf{q}_{\|}^{\prime}, z^{\prime}\right)\right\rangle= & 2 \widetilde{S} C_{z z}\left(\omega, \mathbf{q}_{\|}, z, z^{\prime}\right)(2 \pi)^{3} \\
& \times \delta\left(\omega-\omega^{\prime}\right) \delta\left(\mathbf{q}_{\|}-\mathbf{q}_{\|}^{\prime}\right),
\end{aligned}
$$

with

$$
\begin{aligned}
C_{z z}\left(\omega, \mathbf{q}_{\|}, z_{1}, z_{2}\right)= & \left(z_{1}^{2}-1\right)^{2}\left(z_{2}^{2}-1\right)^{2} C_{I J}\left(\mathbf{q}_{\|}\right) z_{1}^{N} z_{2}^{M} X_{N I}^{-*^{*}} \\
& \times\left(\omega, \mathbf{q}_{\|}\right) X_{M J}^{-1}\left(\omega, \mathbf{q}_{\|}\right),
\end{aligned}
$$

where $X_{N I}^{-1}\left(\omega, \mathbf{q}_{\|}\right)$are the elements of the inverse of the matrix defined in Eq. (34), and where summation over repeated indices is understood. The dynamics of the correlations can be studied on the basis of Eq. (38). However, for the purpose of the present paper, i.e., to determine the amplitude of the nonequilibrium transverse-velocity fluctuations, we need only

$$
C_{z z}\left(\mathbf{q}_{\|}\right)=\frac{1}{2 \pi} \int_{-\infty}^{\infty} d \omega \frac{1}{2} \int_{-1}^{1} d z_{1} \int_{-1}^{1} d z_{2} C_{z z}\left(\omega, \mathbf{q}_{\|}, z_{1}, z_{2}\right),
$$

which represents the equal-time autocorrelation function of the velocity-fluctuation component in the direction of the shear gradient, averaged over the thickness of the layer. In other words, it is the amplitude of the fluctuations of the vertical velocity with a given horizontal wave vector $\mathbf{q}_{\|}$ (again, averaged over the thickness of the layer). This quantity is related to the concept of energy amplification, 
discussed by other authors [25]. Then, substituting all the relevant information into Eq. (39), and performing all integrals, some lengthy but otherwise straightforward calculations allow us to display $C_{z z}\left(\mathbf{q}_{\|}\right)$in a way similar to Eq. (24) for the "bulk" fluctuations, i.e., as an equilibrium contribution plus some nonequilibrium enhancement:

$$
C_{z z}\left(\mathbf{q}_{\|}\right)=C_{z z}^{(\mathrm{E})}\left(\mathbf{q}_{\|}\right)\left\{1+\widetilde{C}_{z z}^{(\mathrm{NE})}\left(\mathbf{q}_{\|}\right)\right\}
$$

where

$$
C_{z z}^{(\mathrm{E})}\left(\mathbf{q}_{\|}\right)=\frac{7}{10} \frac{q_{\|}^{2}}{q_{\|}^{2}+3} \tilde{S} \mathrm{Re},
$$

with $q_{x}=q_{\|} \cos \phi$. Equations (41a) and (41b) represent the final explicit expression in our second-order Galerkin approximation for the vertical-velocity fluctuations with a given wave vector $\mathbf{q}_{\|}$in the plane perpendicular to the shear gradient. If the wave vector is in the horizontal $x y$ plane, the relevant transverse-velocity component affected by the shear corresponds to the velocity component $\delta v_{z}$. Hence, we conclude that Eqs. (41a) and (41b) represent the amplitude of transverse-velocity fluctuations as a function of $\mathbf{q}_{\|}$. To obtain Eqs. (41a) and (41b), the transverse-velocity fluctuations with a given wave vector $\mathbf{q}_{\|}$at a given time $t, \delta v_{z}\left(\mathbf{q}_{\|}, z, t\right)$, were first averaged over the height of the liquid layer and then averaged over the probability distribution of the fluctuations themselves.

Equations (41a) and (41b) incorporate the effects of confinement and it is interesting to compare them with the results, obtained in Sec. III, for the velocity fluctuations in the absence of any boundary conditions. For such a comparison we need to consider the results obtained in Sec. III for the transverse-velocity fluctuations by taking $q_{z}=0$ or, equivalently, $\theta=\pi / 2$. For instance, Eq. (41a) for the equilibrium contribution $C_{z z}^{(\mathrm{E})}$ to the transverse-velocity fluctuations in our Galerkin approximation needs to be compared with the result given by Eq. (25) with $q_{z}=0$. We then observe that, except for a function that rapidly approaches unity as the wave number $q_{\|}$increases, our Galerkin approximation yields an amplitude for the equilibrium correlation function that is $30 \%$ less than the exact value $\tilde{S}$ Re in Eq. (25). We consider this an acceptable result, since it is numerically comparable to what we have obtained in similar calculations for thermal fluctuations in the case of liquid layers subjected to a steady temperature gradient $[4,32]$.

A comparison between the nonequilibrium enhancement of the transverse-velocity fluctuations with and without the effect of boundary conditions is shown in Fig. 1 for two values of the Reynolds number. The top panel corresponds to $\mathrm{Re}=20$ and the bottom panel to $\mathrm{Re}=300$. These are realistic values for the Reynolds number, since in practice the flow is expected to remain laminar for Reynolds numbers up to about $\mathrm{Re}=350[17,33]$. The figures show the amplitude of the nonequilibrium transverse-velocity fluctuations as a function of the wave number $q_{\|}$(with the azimuthal horizontal angle $\phi=0$, i.e., for $\mathbf{q}_{\|}$in the direction of the fluid flow). The dashed curves represent the solution in the absence of any boundary conditions as given by Eq. (26) with $q_{z}=0$. In the absence of boundary conditions, the amplitude exhibits a crossover from a $q_{\|}^{-4}$ to a $q_{\|}^{-4 / 3}$ wave-number dependence as was discussed in Sec. III. The solid curves represent our solution, given by Eq. (41b), that includes the effects of the boundary conditions due to the finite height of the liquid layer. It can be seen that the dashed and solid curves have a common asymptotic behavior for large $q_{\|}$, while the behavior for intermediate and small wave numbers is completely different. From Eq. (41b) we can readily deduce the asymptotic behavior of the nonequilibrium enhancement of the fluctuations for small and large wave numbers in a confined liquid layer:

$$
\begin{gathered}
\widetilde{C}_{z z}^{(\mathrm{NE})}(\mathbf{q}) \stackrel{q \rightarrow 0}{\rightarrow} \frac{2 \mathrm{Re}^{2}}{2079} q_{\|}^{2} \cos ^{2} \phi+O\left(q_{\|}^{4}\right), \\
\widetilde{C}_{z z}^{(\mathrm{NE})}(\mathbf{q}) \stackrel{q \rightarrow \infty}{\rightarrow} \frac{\operatorname{Re}^{2}}{2 q_{\|}^{4}} \cos ^{2} \phi+O\left(q_{\|}^{-6}\right)
\end{gathered}
$$

It is interesting to note that the asymptotic behavior for large $q_{\|}$obtained from the Galerkin approximation exactly reproduces the bulk behavior: Eq. (27b) with $q_{z}=0$. This confirms the goodness of the Galerkin approximation adopted in this investigation for the nonequilibrium fluctuations.

The intensity of the nonequilibrium enhancement of the fluctuations exhibits a crossover from a $q_{\|}^{-4}$ to a $q_{\|}^{2}$ wavenumber dependence, leading to a maximum at an intermediate value $q_{\| m}$ of $q_{\|}$. It follows from Eq. (41b) that $q_{\| m}$ depends on the Reynolds number and on the azimuthal angle through the combination $\mathrm{Re} \cos \phi$. In the limit $\mathrm{Re} \cos \phi$ $\rightarrow 0$ the position of the maximum goes to $q_{\| m} \simeq 2.123$, while for large Re $\cos \phi$ the location of the maximum goes to zero as $q_{\| m} \simeq 9.397(\operatorname{Re} \cos \phi)^{-1 / 2}$. We show in Fig. 2 a plot of the position of the maximum enhancement as a function of Re $\cos \phi$, where the features mentioned above can readily be observed.

A final observation to be mentioned is the presence of an apparent intermediate region between the large and small 


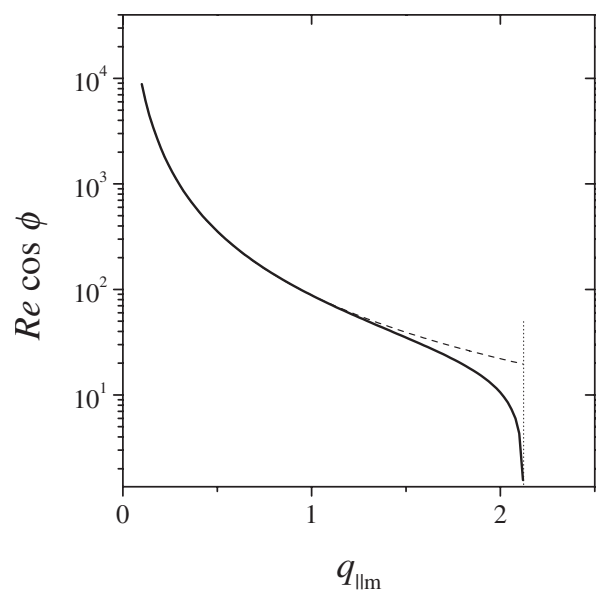

FIG. 2. Wave number $q_{\| m}$ corresponding to the maximum nonequilibrium enhancement of the transverse-velocity fluctuations (horizontal axis) as a function of $\operatorname{Re} \cos \phi$ (vertical axis). In the limit Re $\cos \phi \rightarrow 0$ the location of the maximum approaches $q_{\| m}$ $\simeq 2.123$. The dashed line indicates the asymptotic behavior for large $\operatorname{Re} \cos \phi \rightarrow 0$ (see text)

limiting $q_{\|}$behaviors in Fig. 1, where it seems that the enhancement of the nonequilibrium fluctuations follows neither a $q_{\|}^{-4}$ nor a $q_{\|}^{2}$ wave-number dependence. This phenomenon is especially evident for larger Reynolds number. We suspect that this intermediate behavior could be a residual of the $q_{\|}^{-4 / 3}$ wave-number dependence predicted by other investigators $[11,12]$. However, to arrive at a more definite conclusion we shall need to pursue more carefully an exact numerical computation of the nonequilibrium enhancement of the fluctuations, so as to exclude the possibility that it could be an artifact of our Galerkin-approximation procedure.

\section{DISCUSSION}

In this paper we have considered velocity fluctuations in a liquid layer under steady shear between two plane horizontal boundaries. Specifically, we have evaluated the fluctuations of the vertical velocity component. We have shown that these fluctuations can be determined from a stochastic version of the Orr-Sommerfeld Eq. (8). In the absence of any boundary conditions we have recovered the observations of previous investigators [6-12] about the long-ranged nature of the nonequilibrium fluctuations. The range of these fluctuations is highly anisotropic. For a wave vector $\mathbf{q}_{\|}$in the plane perpendicular to the vertical shear gradient, the fluctuations in the vertical velocity can be fully identified with the transversevelocity fluctuations. We find that the Fourier transform of the nonequilibrium enhancement of these transverse-velocity fluctuations in a liquid layer with finite height exhibits a crossover from a $q_{\|}^{-4}$ wave-number dependence for larger $q_{\|}$ to a $q_{\|}^{2}$ wave-number dependence for small $q_{\|}$with a maximum enhancement at an intermediate wave number $q_{\| m}$. As was shown in Fig. 1, for larger wave numbers the intensity of the nonequilibrium fluctuations continues to vary with $q_{\|}^{-4}$ and is not affected by the boundary conditions, but for smaller wave numbers the crossover behavior is profoundly different from that for the fluctuations predicted in the absence of boundary conditions.

It is interesting to note that the mathematical dependence of the intensity of the nonequilibrium transverse-velocity fluctuations in a liquid layer under steady shear on the wave number is similar to that found for thermal fluctuations in a liquid layer in a stationary temperature gradient, where the intensity of the nonequilibrium temperature fluctuations [5] and the nonequilibrium concentration fluctuations [34] also exhibit a crossover from a $q_{\|}^{-4}$ to a $q_{\|}^{2}$ wave-number dependence. However, there are some physical differences. First, in the case of a stationary temperature gradient, the nonequilibrium enhancement is very large, covering many orders of magnitude $[5,35]$. On the other hand, in the case of steady shear the enhancement is only a factor of 10 or so for Reynolds numbers corresponding to laminar flow, as can be seen from Fig. 1. Second, in the case of a temperature gradient, the intensity of the fluctuations not only increases with the appropriate control parameter, namely, the Rayleigh number $\mathrm{Ra}$, but it diverges at a critical value of the Rayleigh number corresponding to the onset of convection [5,29]. In the case of steady shear, the intensity of the transverse-velocity fluctuations does increase with the Reynolds number, approximately as $\mathrm{Re}^{2}$, but remains finite for any value of the Reynolds number. Hence, unlike the case of a stationary temperature gradient, the nonequilibrium enhancement of the fluctuations in a liquid under steady shear does not lead to any hydrodynamic instability, at least not at the linear fluctuating-hydrodynamics level considered in this paper, in agreement with the observation of previous investigators $[9,11,12]$.

In principle, there is also a connection between the nonequilibrium velocity fluctuations considered in this paper and the concepts of "energy amplification," "stochastic forcing," and "transient growth" in the fluid-mechanics literature $[18,25,36,37]$. The difference is that in the fluid-mechanics literature one tries to determine the nonequilibrium enhancement of externally imposed noise, while we are dealing with the nonequilibrium enhancement of thermal noise. For a more complete treatment of the connection of these concepts with our approach for dealing with nonequilibrium fluctuations, we shall need to consider not only the fluctuations in the velocity component parallel to the shear gradient, but also those of the other components of the velocity. We hope to address this issue in the future by complementing the solution of the stochastic Orr-Sommerfeld equation with that of the stochastic Squire equation (9).

As extensively discussed elsewhere [2], mode coupling always causes the presence of long-ranged fluctuations in liquids in nonequilibrium steady states. The primary conclusion of the present paper is that boundary conditions drastically modify not only the long-ranged nonequilibrium fluctuations in a liquid layer under a stationary temperature gradient, but also the long-ranged fluctuations in a liquid layer under steady shear. In this connection we note that Wada [38] has predicted a crossover from a $q_{\|}^{-4}$ to a $q^{-4 / 3}$ wave-number dependence of the intensity of concentration fluctuations in a binary liquid mixture subjected to a concentration gradient in uniform shear flow. In this case also one must expect that this crossover behavior will be severely affected by finite-size effects [39]. 


\section{ACKNOWLEDGMENTS}

We have greatly appreciated stimulating discussions with James W. Dufty, James F. Lutsko, and Bruno Eckhardt on the topic of this research. We are also indebted to the Spanish Ministerio de Educación y Ciencia for supporting J.V.S. during a sabbatical leave in Madrid, where this work was completed.
[1] J. R. Dorfman, T. R. Kirkpatrick, and J. V. Sengers, Annu. Rev. Phys. Chem. 45, 213 (1994).

[2] J. M. Ortiz de Zárate and J. V. Sengers, Hydrodynamic Fluctuations in Fluids and Fluid Mixtures (Elsevier, Amsterdam, 2006).

[3] J. V. Sengers and J. M. Ortiz de Zárate, J. Non-Equilib. Thermodyn. 32, 319 (2007).

[4] J. M. Ortiz de Zárate and J. V. Sengers, Physica A 300, 25 (2001).

[5] J. M. Ortiz de Zárate and J. V. Sengers, Phys. Rev. E 66, 036305 (2002).

[6] J. Machta, I. Oppenheim, and I. Procaccia, Phys. Rev. A 22, 2809 (1980).

[7] A. M. S. Tremblay, M. Arai, and E. D. Siggia, Phys. Rev. A 23, 1451 (1981).

[8] J. Machta and I. Oppenheim, Physica A 112, 361 (1982).

[9] J. Lutsko and J. W. Dufty, Phys. Rev. A 32, 3040 (1985).

[10] J. W. Dufty and J. Lutsko, in Recent Developments in Nonequilibrium Thermodynamics: Fluids and Related Topics, edited by J. Casas-Vázquez, D. Jou, and J. M. Rubí, Lecture Notes in Physics Vol. 253 (Springer, Berlin, 1986), pp. 47-84.

[11] J. F. Lutsko and J. W. Dufty, Phys. Rev. E 66, 041206 (2002)

[12] H. Wada and S. I. Sasa, Phys. Rev. E 67, 065302(R) (2003).

[13] J. M. Ortiz de Zárate and J. V. Sengers, J. Stat. Phys. 115, 1341 (2004).

[14] D. Ronis and I. Procaccia, Phys. Rev. A 26, 1812 (1982).

[15] T. R. Kirkpatrick, E. G. D. Cohen, and J. R. Dorfman, Phys. Rev. A 26, 995 (1982).

[16] P. G. Drazin and W. H. Reid, Hydrodynamic Stability, 2nd ed. (Cambridge University Press, Cambridge, U.K., 2004).

[17] P. J. Schmid and D. S. Henningson, Stability and Transition in Shear Flows (Springer, Berlin, 2001).

[18] B. Eckhardt and R. Pandit, Eur. Phys. J. B 33, 373 (2003).

[19] L. D. Landau and E. M. Lifshitz, Fluid Mechanics (Pergamon,
London, 1959, 2nd revised English version 1987).

[20] R. Schmitz and E. G. D. Cohen, J. Stat. Phys. 39, 285 (1985).

[21] B. M. Law and J. V. Sengers, J. Stat. Phys. 57, 531 (1989).

[22] J. P. Hansen and I. R. McDonald, Theory of Simple Liquids, 2nd ed. (Academic Press, London, 1986).

[23] J. P. Boon and S. Yip, Molecular Hydrodynamics (McGrawHill, New York, 1980/Dover, New York, 1991).

[24] B. J. Berne and R. Pecora, Dynamic Light Scattering (Wiley, New York, 1976/Dover, New York, 2000).

[25] B. Bamieh and M. Dahleh, Phys. Fluids 13, 3258 (2001).

[26] B. M. Law and J. C. Nieuwoudt, Phys. Rev. A 40, 3880 (1989).

[27] T. R. Kirkpatrick, D. Belitz, and J. V. Sengers, J. Stat. Phys. 109, 373 (2002).

[28] J. M. Ortiz de Zárate, R. Pérez Cordón, and J. V. Sengers, Physica A 291, 113 (2001).

[29] J. M. Ortiz de Zárate, J. A. Fornés, and J. V. Sengers, Phys. Rev. E 74, 046305 (2006).

[30] R. Schmitz and E. G. D. Cohen, J. Stat. Phys. 40, 431 (1985).

[31] A. V. Dyachenko and A. A. Shkalikov, Funct. Anal. Appl. 36, 228 (2002).

[32] J. M. Ortiz de Zárate and L. Muñoz Redondo, Eur. Phys. J. B 21, 135 (2001).

[33] L. N. Trefethen, A. Trefethen, S. C. Reddy, and T. A. Driscoll, Science 261, 578 (1993).

[34] J. V. Sengers and J. M. Ortiz de Zárate, Rev. Mex. Fis. 48, Suppl. 1, 14 (2002).

[35] A. Vailati and M. Giglio, Phys. Rev. Lett. 77, 1484 (1996).

[36] B. F. Farrell and P. J. Ioannou, Phys. Fluids A 5, 2600 (1993).

[37] D. Biau and A. Bottaro, Phys. Fluids 16, 3515 (2004).

[38] H. Wada, Phys. Rev. E 69, 031202 (2004).

[39] J. M. Ortiz de Zárate and J. V. Sengers, Phys. Rev. E 73, 013201 (2006). 